\title{
Different MRI Sequences and Spectrum of Findings in Pediatric Brain Infections in North Indian Population
}

\author{
Shagufta Wahab ${ }^{1}$, Prabhash Chandra Jha ${ }^{2}$, Rizwan Ahmad Khan ${ }^{3}$, Zeeba Zakaur Rab ${ }^{4}$, Kumari Chitra ${ }^{5}$ \\ ${ }^{1}$ Associate Professor, Department of Radiodiagnosis, JNMCH, AMU, Aligarh, ${ }^{2}$ Junior Resident, Department of Radiodiagnosis, \\ JNMCH, AMU, Aligarh, ${ }^{3}$ Associate Professor, Department of Pediatric Surgery, JNMCH, AMU, Aligarh, ${ }^{4}$ Professor, Department \\ of Pediatrics, JNMCH, AMU, Aligarh, ${ }^{5}$ Junior Resident, Department of Anaesthesiology, JNMCH, AMU, Aligarh, India
}

Corresponding author: Dr. Rizwan A. Khan, Kashan-e-Wahab, Street No 4, Iqra Colony, Aligarh, U.P., India

DOI: http://dx.doi.org/10.21276/ijcmsr.2019.4.4.29

How to cite this article: Shagufta Wahab, Prabhash Chandra Jha, Rizwan Ahmad Khan, Zeeba Zakaur Rab, Kumari Chitra. Different MRI sequences and spectrum of findings in pediatric brain infections in North Indian population. International Journal of Contemporary Medicine Surgery and Radiology. 2019;4(4):D124-D127.

\section{A B S T R A C T}

Introduction: Pediatric brain infections pose a significant challenge to health professionals in developing countries like India due to the high mortality and morbidity associated with them and delays in establishing an early diagnosis. This study was planned to establish the role of MRI and evaluate the spectrum of imaging findings in this group of patients of North India. Material and Methods: This study was performed on 70 children who were less than 14 years of age and had presenting complaints suggestive of brain infection from Nov 2016 to Nov 2018 at JN Medical College and Hospital, Aligarh. MRI was performed on 1.5 Tesla Siemens MAGNATOM Avantofit withT1 weighted, T2 weighted, Fluid Attenuated Inversion Recovery (FLAIR), Post Contrast T1 and newer sequences like Susceptibility Weighted Imaging (SWI), Constructive Interference in Steady State (CISS), Diffusion Weighted Imaging (DWI) and MR Spectroscopy.

Results: Out of 70 patients evaluated -One was of congenital CMV infection ( 1.4\%), 11patients of acute pyogenic meningitis ( $15.7 \%)$ and, 29 patients with tubercular meningitis and tuberculoma ( 41.4\%). 21 patients of Neurocysticercosis ( 30\%) and 8 of viral encephalitis out of which 2 patients were positive of Herpes simplex virus 1 infection( 2.9\%) and 6 patients diagnosed with Japanese encephalitis ( $8.6 \%)$.CISS sequence helped in identifying the scolex in neurocysticercosis, SWI was useful for evaluation of calcification in granulomatous lesions and hemorrhagic foci in Japanese encephalitis. Post contrast T1 weighted sequence was useful for accurate delineation of size of ring enhancing lesions and their follow up. Diffusion weighted Imaging and MR spectroscopy too had their specific utility in establishing the differential diagnosis.

Conclusion: Combining the conventional MRI sequences with the newer ones gives a wide spectrum of imaging findings in pediatric brain infections with specific features of different etiology on different sequences thus establishing accurate diagnosis in majority of the cases or at least narrows down the differential for early management.

Keywords: MRI Sequences, Pediatric, Meningitis, Tuberculoma, Neurocysticercosis, Encephalitis

\section{INTRODUCTION}

Infection of the central nervous system (CNS) is one of the most common causes of mortality and morbidity in children. In general, viral infections are most prevalent followed by bacterial, fungal and parasitic infections. The incidence of acute bacterial meningitis is 2.2 cases for 100,000 persons per year in the world. Meningitis caused by pneumococcus has a risk of mortality of $20 \%$ and a high rate of sequelae 30 to $50 \% .{ }^{1}$ Meningoencephalitis has a risk 10 to $12 \%$ mortality even with todays treatment options available. The main problem is of early diagnosis, with an identified aetiology in only 52 to $63 \%$ of case. ${ }^{1}$ According to Indian records 0.5 to $2.6 \%$ of hospital admissions are of acute bacterial meningitis (ABM), the case fatality rate being 20 to $30 \%$ due to delayed presentation of the patients.

Despite different causative agents, most patients with brain infection have similar clinical signs and symptoms like headache, altered consciousness, nausea, vomiting, irritability, fever, neck pain or rigidity, seizures and/or focal neurologic deficits. Clinical evaluation and lab investigations previously used to be the basis for starting treatment but MRI is now the imaging modality of choice for evaluation of the extent and complications of these infections. MRI not only helps in differentiating brain infections from other pathologies like tumors, vascular insults, white matter disorders, but it also helps in differentiating the different etiologies of brain infections like viral encephalitis, tubercular infection, neurocysticercosis or meningitis. The purpose of this study was to evaluate the spectrum of MRI findings on different sequences in Indian children with suspected brain infections and evaluate the extended role of MRI in establishing the final diagnosis.

\section{MATERIAL AND METHODS}

This study was performed on 70 children who were of 14 years of age or less with presenting complaints suggestive of brain infection from Nov 2016 to Nov 2018. Detailed clinical 
history, physical examination, blood and CSF examination was done. This was followed by MRI performed on 1.5 Tesla Siemens MAGNATOM Avantofit with syngo MR 11 software. The detailed methodology pertained to various MRI sequences and these include: T1 Weighted sagittal, axial and coronal images: TR/TE (repetition time/ time to echo) - 650 to $1700 \mathrm{~ms} / 3$ to $17 \mathrm{~ms}$, inversion time $900 \mathrm{~ms}\}$, turbo Spin Echo (TSE) mode with slice thickness 3-4mm with gap of $1 \mathrm{~mm}$ and FOV $20 \mathrm{~cm}$; T2 weighted imaging sequence TR/TE (4050 to $4500 \mathrm{~ms} / 90$ to101 ms), TSE mode with slice thickness $3-4 \mathrm{~mm}$ with gap of $1 \mathrm{~mm}$ and FOV $20 \mathrm{~cm}$; FLAIR sequence $\mathrm{TR} / \mathrm{TE}=9000 \mathrm{~ms} / 90 \mathrm{~ms}$; inversion time, $2500 \mathrm{~ms}$. TSE mode with slice thickness 3-4mm with gap of $1 \mathrm{mmFOV} 20 \mathrm{~cm}$; Susceptibility weighted Imaging (SWI); contrast enhanced T1 weighted imaging sequence sagittal, axial and coronal sequence; CISS sequence:- (TR/TE $=5.44$ $\mathrm{ms} / 2.44 \mathrm{~ms}$ ) with slice thickness was $0.5 \mathrm{~mm}$ with interslice gap of $20 \%$ with matrix size of 256x256 mm; Diffusion weighted imaging (DWI) and apparent diffusion coefficient (ADC) imaging were performed using echo planar imaging sequence with $\mathrm{TR} / \mathrm{TE}=3100$ to $3500 \mathrm{~ms} / 77$ to $109 \mathrm{~ms}$ with field of view $(\mathrm{FOV})=23 \mathrm{~cm} \times 23 \mathrm{~cm}$, number of excitations $=3$, slice thickness $=5 \mathrm{~mm}$, inter-slice gap $=1.5 \mathrm{~mm}$, matrix size $=260 \times 260$, Diffusion sensitizing gradients were applied along the three planes with at $\mathrm{b}=0, \mathrm{~b}=500$, and $\mathrm{b}=1000 \mathrm{~s} /$ $\mathrm{mm}$; MR Spectroscopy for volume of interest from the lesion was selected on SE T2 weighted imaging sequence, Multi voxel spectroscopy (MVS) was performed in these lesions and these studies were performed at TE $135 \mathrm{~ms}$ and $270 \mathrm{~ms}$; Contrast-enhanced FLAIR sequence in axial, coronal and sagittal plane were done, whenever needed.

After obtaining various sequences, findings were tabulated and findings on imaging were compared on different sequences as well as correlated with final diagnosis.

\section{RESULTS}

Total seventy patients $0-14$ years of age with clinical sign and symptoms of brain infections were examined in this study with MRI evaluation from Nov 2016 to Nov 2018.

There were $38(54.3 \%)$ females while male patients 32 (45.7\%). Older children in 10-14 year age group were the most commonly affected (42.86\%), followed by 5 to 9 years $(30 \%)$ and the least common affected age group was neonates (2.85\%). Most of the patients presented with fever and altered sensorium and seizures with a few having neurological deficits.

Final diagnosis in our study group included -One patient with congenital CMV infection $(\sim 1.4 \%), 11$ patients with

\begin{tabular}{|l|c|c|}
\hline Diagnosis & $\begin{array}{c}\text { No. of } \\
\text { cases }\end{array}$ & Percentage \\
\hline Congenital CMV infection & 1 & $1.4 \%$ \\
\hline Acute pyogenic meningitis & 11 & $15.7 \%$ \\
\hline Tubercular meningitis and Tuberculoma & 29 & $41.4 \%$ \\
\hline Neurocysticercosis & 21 & $30 \%$ \\
\hline Japanese Encephalitis & 6 & $8.6 \%$ \\
\hline HSV & 2 & $2.9 \%$ \\
\hline Table-1: Spectrum of disease among paediatric brain infection \\
\hline
\end{tabular}

acute pyogenic meningitis ( 15.7\%) and, 29 patients with tubercular meningitis and tuberculoma ( 41.4\%). Twenty one patients were diagnosed as neurocysticercosis ( 30\%), 8 patients as viral encephalitis out of which 2 patients were positive of Herpes simplex virus 1 infection ( 2.9\%) and 6 patients diagnosed with Japanese encephalitis ( 8.6\%)[Table 1]. Total number of patients with TBM was 29 out of which tuberculoma was seen in 17 cases. All the cases presented with ring like enhancing lesions. Nodular enhancement was also seen in 6 cases in addition to the ring enhancing lesions. Among the diagnosed cases of tuberculoma, single lesion was seen in 6 cases, multiple conglomerated lesions were seen in 9 cases and multiple isolated lesions noted in 2 cases.

Neurocysticercosis (NCC) was seen in 21 cases. Eight patients presented with single lesions whereas 13 patients presented with multiple lesions. Intraparenchymal forms of NCC were seen in all the cases with 12 cases having subarachnoid location as well. Scolex was identified in 6 cases. MR Spectroscopy showed choline and lactate peak and reduced NAA peak [Fig.1a and b].

Susceptibility Weighted Imaging (SWI imaging) helped to identify calcified lesions which were seen in 8 cases. All the lesions were hypo to isointense on $\mathrm{T} 1$ weighted images and 12 cases were hyperintense on T2 weighted image. Infections with the ring enhancing lesion were tuberculoma, neurocysticercosis and brain abscess. The characterization of lesions was done on the basis of various appearance on T1 weighted, T2 weighted imaging, diffusion restriction on diffusion weighted imaging and MR spectroscopy. Total number of cases encounter with ring enhancing lesion was 39. The number of neurocysticercosis patients was 21 followed by tuberculoma 17 , and abscess, which was seen in only one patient.

There were 29 patients who were diagnosed with tubercular infection. It presented as ring enhancing lesion in 17 patients, post contrast meningeal enhancement was noted in 20 patients, basal exudates seen in 16 patients and nodular lesion was seen in 6 patients.

Complications were noted in 12 patients, hydrocephalus was seen in 9 patients and variable sized infarcts in 3 patients and hydrocephalus with infarcts in 2 patients [Fig.2a and b]

Meningeal enhancement was noted in all 11 patients of acute pyogenic meningitis. Four patients had complications in the form of hydrocephalus and one patient had infarction.

Only 8 patients were diagnosed with encephalitis, in which 6 cases were Japanese encephalitis and 2 cases were herpes simplex encephalitis. Out of 6 cases- high signal intensity on T2WI and FLAIR sequence in bilateral basal ganglia region was seen in 4 cases, brain stem in 3 cases and cortical involvement in 3 . On diffusion weighted imaging there was restriction noted in the involved region in all 4 cases, which was seen on T2Weighted Imaging and one more case which was not seen on T2 Weighted Imaging.

Blooming on susceptibility weighted imaging sequence was seen in 2 cases involving bilateral basal ganglia region, which was suggestive of hemorrhage. No meningeal enhancement was seen. The final diagnosis was confirmed on serology by the presence of IgM antibody in the CSF. Herpes simplex encephalitis was seen in 2 cases. On T2WI and FLAIR 
sequences symmetrical hyperintensity was noted in both frontal and temporal lobes in both the cases. On diffusion weighted imaging restriction of diffusion was seen in both the cases and one of the lesions appeared larger compared to the area of involvement seen on conventional T2Weighted and FLAIR sequence. Blooming was not seen on SWI sequences, which ruled out any hemorrhage. On post contrast imaging there was mild meningeal enhancement of the leptomeninges overlying bilateral temporal lobes with mild gyral enhancement. The confirmation was done by DNAPCR analysis of CSF which showed positive for HSV type1 infection [Fig-3 a and b].

\section{DISCUSSION}

Seventy patients were evaluated, whose age group ranged from 0 to 14years. The highest incidence of brain infections was found in 10 to 14 years age group accounting for $43 \%$ of cases and least was seen in neonatal age group constituting $2.9 \%$ of cases. However the incidence of acute pyogenic meningitis was quite high in Infants as compared to older children which are consistent with other previous studies. ${ }^{2}$ There was slightly more incidence of brain infection in the female child as compared to male child 38 (53.5\%) versus 32 (46.5\%). This finding is different from previous studies on Indian population. ${ }^{3}$ We found in our study that the incidence of tubercular meningitis was comparatively higher than the acute pyogenic meningitis. Only one case of congenital cytomegalovirus infection was seen in our study. Imaging features seen were microcephaly, enlargement of temporal horns and periventricular calcification. However there was no evidence ofcortical abnormalities in the form of agyria or pachygyria. Certain imaging features may indicate future neurologic deficits in symptomatic infants. ${ }^{4}$ Acquired CMV infection is potentially fatal in immuno-compromised patients. The imaging findings of acquired CMV infection are nonspecific and in such cases serologic examination for confirmation of CMV infection is required.

Tubercular meningitis with tuberculomas in 29 cases (41.4\%) was the most common pathology followed by 21 cases of NCC (30\%), 11 cases of acute pyogenic meningitis (15.7\%), 6 cases of Japanese encephalitis (8.6\%), 2 cases of HSE (2.9\%) and least common was 1 case of congenital CMV infection (1.4\%). The higher incidence of tuberculosis is probably due to the higher prevalence of tuberculosis in India. Most of the tubercular infections were associated with features of meningitis like meningeal enhancement and basal exudates. It is difficult to differentiate between tuberculoma and neurocysticercosis, in such situations, the use of advanced sequences of MR imaging becomes helpful like diffusion weighted imaging and MR spectroscopy. Diffusion weighted imaging can be used to differentiate between tuberculoma, tubercular abscess and neurocysticercosis. ${ }^{5}$ For the characterization of tubercular ring, use of MR spectroscopy was performed, which showed lipid metabolite peak in 12 cases, helping to differentiate from neurocysticercosis and other ring enhancing lesion with associated decrease inpeak of NAA with and creatinine and elevated choline. By the use of MR spectroscopy it was possible to differentiate between abscess and tuberculoma because in abscess there was lactate peak, while in tuberculoma lipid peak was seen. ${ }^{6}$ Jayasundar et al in 1999 evaluated different groups of patients with granulomatous lesion and studied about the MR spectroscopy findings. There was increase in lipid peak in $80 \%$ of cases of tuberculoma and in $20 \%$ of neurocysticercosis cases as compared to our study in which approximately $70 \%$ of tuberculoma cases were having increased lipid peak while $16 \%$ of patients of neurocysticercosis had lipid peak. ${ }^{7}$ The different stages of tuberculoma whether it is caseating or non-caseating granuloma, can also be determined by MRI on T2 weighted imaging. ${ }^{5}$ Susceptibility weighted imaging was helpful in the diagnosis of calcified granuloma and follow up of treatment. Post contrast T1 weighted imaging was very helpful in delineating the margin of lesion which is important in accurate assessment of size of the ring enhancing lesion. It also helps to differentiate from the perilesional edema and provides better spatial resolution. In neurocysticercosis, 3D-CISS sequence helped to identify scolex in 6cases. Scolex and cyst wall may not be seen on routine sequences, but a $3 \mathrm{DCISS}$ sequence may demonstrate it due to its higher contrast-to-noise ratio. ${ }^{8}$ We found that cho/ $\mathrm{Cr}$ ratio was less than 1.1 in neurocysticercosis and more than 1.2 in tuberculoma, similar to previous studies emphasizing the additional utility of spectroscopy. ${ }^{7,9}$

In evaluation of viral encephalitis, early detection of lesions or an extensive area of involvement was well demonstrated utilizing Diffusion weighted sequences. The sensitivity of DWI in early diagnosis of viral encephalitis was seen in 2 cases of Japanese Encephalitis and 1 of Herpes encephalitis. Kuker et al in 2004 in their study also established the capability of DWI in early detection and diagnosis of viral encephalitis.

\section{CONCLUSION}

To conclude, MRI remains the gold standard imaging tool for diagnosis and management of paediatrics brain infections. Conventional MRI sequences, post contrast T1 weighted image, fluid attenuated inversion recovery (FLAIR) image aided with newer sequences, constructive interference in steady state (CISS), magnetic resonance spectroscopy (MRS) and diffusion weighted image (DWI) not only help in clearly establishing the differential diagnosis but also in early diagnosis and follow up of treatment and sequelae. A full and updated knowledge and application of the conventional as well as advanced sequences can significantly increase the utility of MRI in improving the prognosis of children with brain infections.

\section{REFERENCES}

1. Sarrazin JL, Bonneville F, Martin-Blondel G.Brain infections. Diagn Interv Imaging. 2012;93(6):473-90.

2. Sheehan JP, Jane JA, Ray DK, Goodkin HP. Brain abscess in children. Neurosurg Focus. 2008;24(6):E6.

3. Garg A, Sharma A, Kumari S, Shandil A. Clinical profile and outcome ofpediatric bacterial meningitis: a prospective study from tertiary institute inNorthern India. Int J Res Med Sci 2018;6:2739-45.

4. Fink KR, Thapa MM, Ishak GE,Pruthi S.Neuroimaging of pediatric central nervous system cytomegalovirus 
infection. Radiographics 2010;30(7):1779-96.

5. Gupta RK, Kohli A, Gaur V et al. MRI of the brain in patients with miliary pulmonary tuberculosis without symptoms or signs of central nervous system involvement. Neuroradiology 1997; 39(5):699-704.

6. Mishra AM, Gupta RK, Jaggi RS, et al. Role of diffusion-weighted imaging and in vivo proton magnetic resonance spectroscopy in the differential diagnosis of ring- enhancing intracranial cystic mass lesions. J Comput Assist Tomogr 2004;28(3):540-547.

7. Jayasundar R, Singh VP, Raghunathan P, Jain K, Banerji AK. Inflammatory granulomas: evaluation with proton MRS. NMR Biomed. 1999;12(3):139-44.

8. Hingwala D, Chatterjee S, Kesavadas C, Thomas B, Kapilamoorthy TR. Application of 3D CISS sequence for problem solving in neuroimaging. Indian J Radiol Imaging. 2011; 21(2):90-97

9. Kuker W, NageleT, Schmidt F et al. Diffusion Weighted MRI in herpes simplex encephalitis: a report of three cases. Neuroradiology 2004; 46(2):122-5.

10. Rath TJ, Hughes M, Arabi M, Shah GV. Imaging of cerebritis, encephalitis, and brain abscess. Neuroimaging Clin N Am. 2012;22(4):585-607.

Source of Support: Nil; Conflict of Interest: None

Submitted: 25-10-2019; Accepted: 16-11-2019; Published online: 19-12-2019 